\title{
Study on microstructure and high-temperature tribological properties of multi-component nitride hard coatings
}

\author{
Baiyang Lou ${ }^{a}$, Xia Zhang ${ }^{b}$, Bin Xuc ${ }^{c}$ Xiao Li ${ }^{d}$ and Jie Jin ${ }^{\mathrm{e}}$
}

Institute of material forming and control engineering, Zhejiang University of Technology, Hangzhou 310014, China

aby00518@163.com, ${ }^{\mathrm{b}} 15264316715 @ 163 . c o m,{ }^{\mathrm{c}}$ lixiaobruce@163.com, ${ }^{\mathrm{d}} \mathrm{xub} @$ zjut.edu.cn jinjie@zjut.edu.cn

Keywords: CrTiAlN, M2 high speed steel, High-temperature tribological properties

Abstract. In this study, CrTiAlN and CrAlN coatings were deposited on the surfaces of M2 high speed steel (HSS) substrates. High temperature ball-on-disc tribometer was used to test the tribological properties of the coatings. The results showed that, the surface micro-morphology of CrTiAlN and CrAIN presented closely packed structure with relatively uniform grains of circular shape and the cross-sectional morphology demonstrated columnar structures. XRD studies revealed that the coatings mainly consist of $\mathrm{CrN}, \mathrm{Cr}$ and $\mathrm{Cr}_{2} \mathrm{~N}$ crystal phases. High temperature wear tests illustrated from room temperature to $600^{\circ} \mathrm{C}$, friction coefficients of CrTiAlN and CrAlN coatings increased at first and then decreased with the temperature elevated and the wear rate of the two coatings increased as the temperature rose. Besides, the wear rate of CrAlN coating was greater than CrTiAlN coating at the same temperature.

\section{Introduction}

M2 high speed steel is a kind of tool steel with high hardness, wear resistance, heat resistance and favorable combining performance of strength and toughness. It has been widely used as cutting tools materials in machining since it was born. With the development of modern cutting technology, the cutting speed is getting faster and faster and the performance requirement of cutting tool is also getting higher and higher. In recent decades, with the development of modern science and technology, research of surface coating technology and materials has made rapid development. Surface coating technology is a cross technology involving materials, chemistry, physics and other subjects. It is an important method for surface modification of materials. It has been widely used in the fields such as semiconductor, instrument, space, nuclear energy and mechanics ${ }^{[1-3]}$. Using the physical, chemical vapor deposition or other methods to form a kind of refractory metal hard coating (such as $\mathrm{CrN}$, TiN, etc.) or nonmetallic compound soft coating (such as $\mathrm{MoS}_{2}$, etc.) with excellent wear-resistant, high temperature resistance or other special properties on the tool and die surfaces has been drawn people's attention $^{[4-6]}$.

Based on the TiN hard coating, CrTiAlN coating and CrAlN coating were prepared by $\mathrm{Al}, \mathrm{Cr}$ and $\mathrm{Ti}$ targets on the M2 high speed steel substrates, and the microstructure of the coating, high temperature friction and wear properties were studied in this work.

\section{Experimental materials and methods}

M2 high speed steel is used as the substrate material (hardness is about 60HRC). In order to ensure the adhesion between the film and substrate, we polished the specimens surface with different grades of water sandpapers and the samples were polished to the surface roughness $\mathrm{Ra}$ is less than or equal to 0.5 um on the polishing machine with diamond polishing slurry. In order to remove the surface oil pollution and other impurities, the polished steel and silicon wafers were ultrasonically cleaned using acetone and ethanol in proper sequence. The cleaned samples were placed in a vacuum oven and stayed to be used in the experiment after they were completely dry. The coating morphology was observed by ¿IGMA type field emission scanning electron microscope (FESEM). The sample which has a scratch 
from the backward to the near surface was cooled down in liquid nitrogen and then broken in half. The cross-sectional morphology of the specimen and the coating thickness were examined by electron microscope. Bruker Nano XFlash Detector 5010 Spectrometer which is the attachment of FESEM was used to determine chemical composition of the coatings. The hardness of the coating was evaluated using the Rockwell hardness tester. In order to reduce the error, each sample was measured 5 times and then we took the average value.

Wear tests of the coated and uncoated specimens were performed on a ball-on-disc tribometer HT-600 under the setting load and rotating speed while the specimen was fixed on the rotating sample table and the experimental data such as testing temperature, load and friction coefficient were collected by the master computer.

The tests were conducted with no lubricant along a circular track of $6 \mathrm{~mm}$ diameter against a $4 \mathrm{~mm}$ diameter WC ball at 350r/min under the normal load of $10 \mathrm{~N}$ in the ambient atmosphere and $50 \%$ relative humidity. Experiments were carried out at room temperature, $200^{\circ} \mathrm{C}, 400^{\circ} \mathrm{Cand}$ $600^{\circ}$ Crespectively.

\section{Experimental results and analysis}

\section{Microstructure and chemical composition of the coatings}

The surface morphology of the composite CrTiAlN coating is shown in Figure 1(a). It can be seen that the surface of the coating presented closely packed structure with relatively uniform grains of circular shape and the average size of the grains was about $150 \mathrm{~nm}$. Fig 1(b) is a cross-sectional morphology of CrTiAlN coating, it is observed that this coating had an analogous columnar morphology and the columnar crystals were arranged densely and performed without obvious pores and defects. In addition, fine crystalline structure can be observed at the region close to the substrate, which ensures a good adhesion between the coating and the substrate.

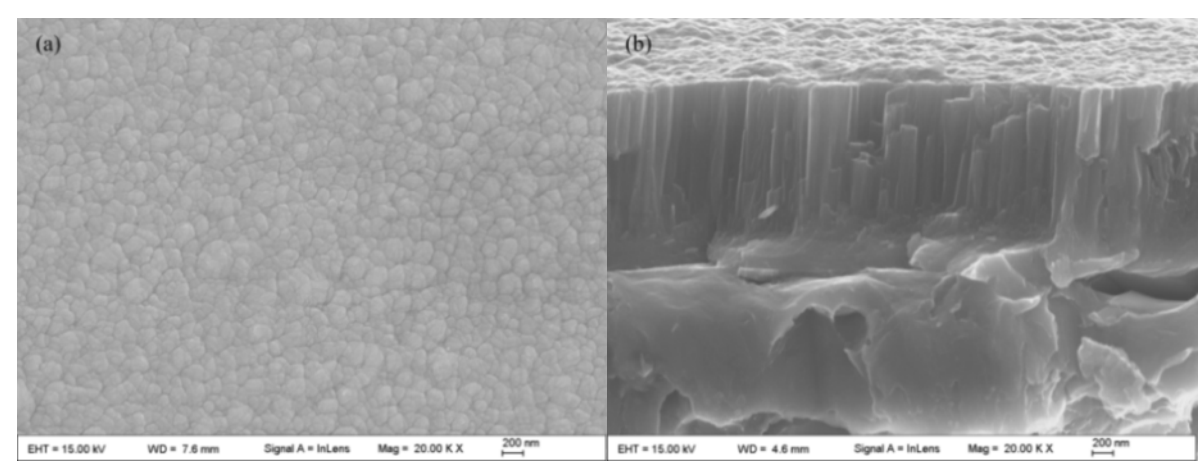

Fig.1 Microstructure of CrTiAlN coating

Figure 2(a) is a surface morphology of CrAIN coating. We can see that the coating surface was granular with closely packed particles. Figure2 (b) is a SEM cross-sectional image of CrAIN coating, which is similar to the CrTiAlN coating and presented obvious columnar dense structure without obvious defects.

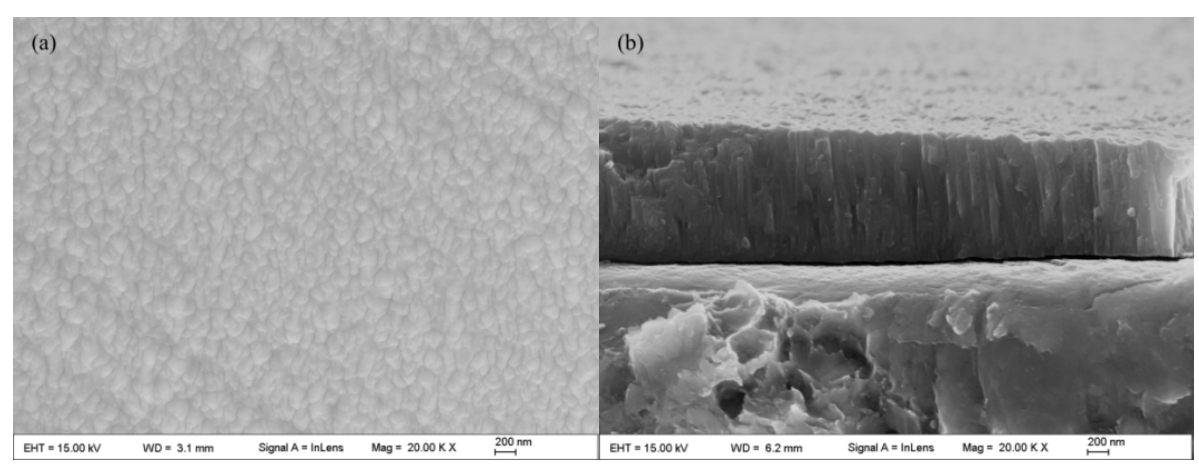

Fig.2 Microstructure of CrAlN coating 


\section{Bonding conditions between the films and substrates}

Fractured morphology of the coatings of CrTiAlN and CrAlN by the Rockwell adhesion tests are shown in Figure 3 and Figure 4. There was only a little spot flaking and circumferential cracks around the indentation in Figure 3(a). Figure 3(b) appeared more flaking, but not throughout the entire indentation. This phenomenon illustrates that the adhesion between CrTiAlN coating and M2 high speed steel is still good even after oxidation at high temperature $600^{\circ} \mathrm{C}$ for one hour. A small amount of radial cracks, circumferential cracks, and point like spalling occurred around the indentation in Figure 4 (a). In Figure 4(b), massive circumferential cracks around the entire indentation were found. This suggests that after high temperature oxidation at $600^{\circ} \mathrm{C}$ for $1 \mathrm{~h}$, there appears massive spalling at the edge of the indentation, which shows that the combination of CrAlN coating and M2 high speed steel substrate has been poor.

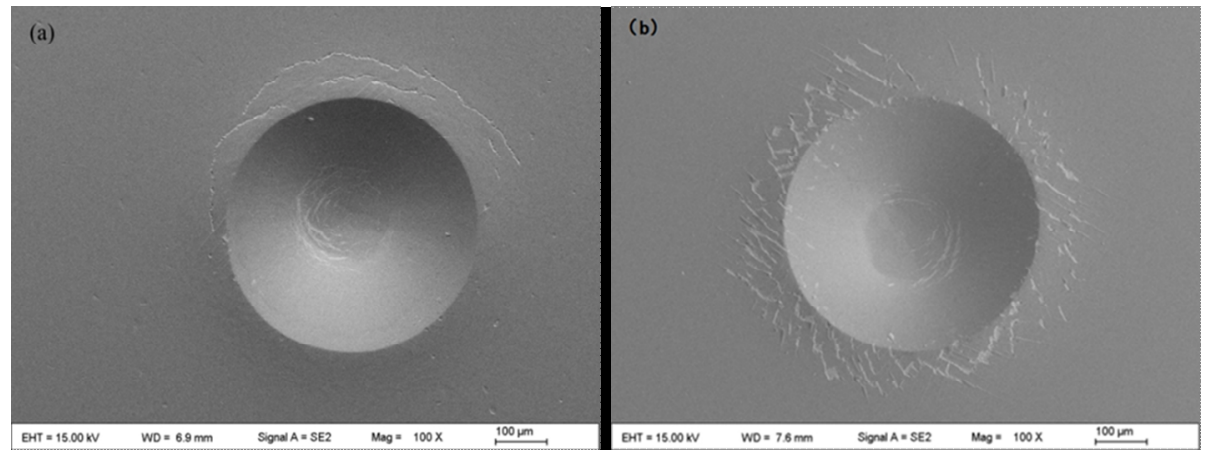

Fig.3 Fractured morphology of CrTiAlN coatings by the Rockwell adhesion tests: (a) $23^{\circ} \mathrm{C}$ and (b) $600^{\circ} \mathrm{C}$

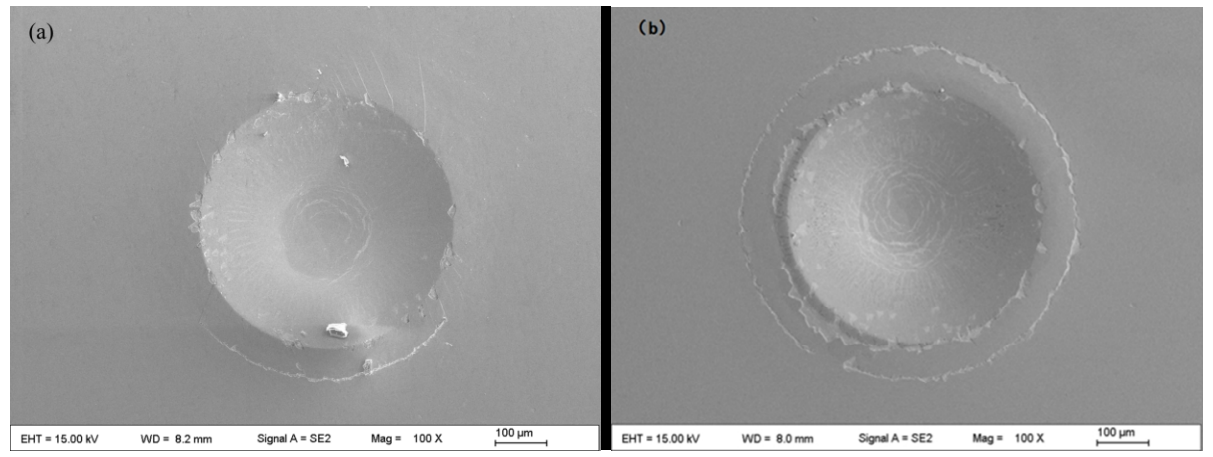

Fig.4 Fractured morphology of CrAlN coatings by the Rockwell adhesion tests: (a) $23^{\circ} \mathrm{C}$ and (b) $600^{\circ} \mathrm{C}$

\section{Analysis of high-temperature tribological behaviors}

Figure 5 shows the friction coefficient is function of time. We can see that temperature has a great influence on the friction coefficient of the coatings. The friction process of the coating had experienced two stages of running and stability. At the running stage, the friction coefficient of the films increased rapidly due to the initial surface of the coating had a certain degree of roughness, as the experiment proceeded, the contiguous surface between the coating and the grinding ball became smooth and the oxides produced by the oxidation on the surface of the coating were beneficial to the wear, so the friction coefficient began to decline. At last, the friction process reached a stable stage when the friction system reached equilibrium.

In General, the friction coefficients of the two coatings both were relatively small at room temperature. But they fluctuated greatly at high temperature, which illustrated that with increase of temperature, the instability factors between the coating and the grinding ball increased. It is obvious that the friction coefficients of the two coatings increased from $23^{\circ} \mathrm{C}$ to $400^{\circ} \mathrm{C}$ but decreased at $600^{\circ} \mathrm{C}$, because the oxide coatings formed on the surface of the films at high temperature were beneficial to the wear. High-temperature tribological properties of CrTiAlN are better than CrAlN. This is due to the addition of Ti element makes it possible to produce more oxides on the surface of substrate at high temperature. 


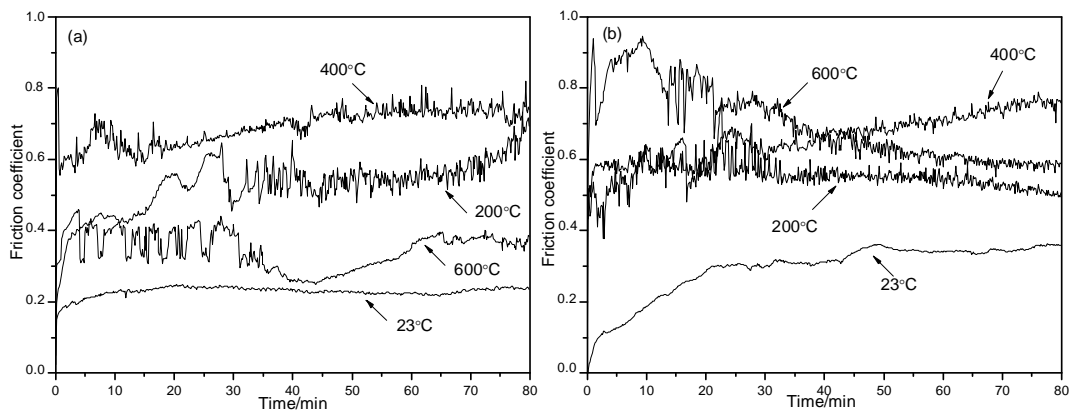

Fig.5 Friction coefficients of CrTiAlN coating (a) and CrAlN coating (b) at different temperatures

\section{Conclusions}

(1)The surface morphology of the CrTiAIN and CrAlN coatings were presented closely packed structure with relatively uniform grains of circular shape. And the average size of the grains for CrTiAlN was about $150 \mathrm{~nm}$. Cross-sectional morphology of the two coatings had an analogous columnar morphology and the columnar crystals were arranged densely and performed with no obvious pores and defects. In addition, the fine crystalline structure can be observed near the substrate area for CrTiAlN which ensures a good adhesion between the coating and substrate.

(2) From room temperature $23^{\circ} \mathrm{C}$ to $400^{\circ} \mathrm{C}$, the friction coefficients of CrTiAlN and CrAlN coatings both increased, but they decreased at $600^{\circ} \mathrm{C}$. It is because that the oxide coatings formed on the surface at high temperature were beneficial to the wear. At room temperature, the friction coefficient curves of the two kinds of coatings were smooth, but they fluctuated greatly at high temperature which was mainly due to the instability factors increased.

\section{Acknowledgement}

The work is granted by Natural Science Foundation of Zhejiang Province (No.Y15E050060) and Zhejiang province science and technology program (No. 2014C31126).

\section{References}

[1] Z.B. Qi, Z.T.Wu, Z.C.Wang, Improved hardness and oxidation resistance for CrAlN hard coatings with Y addition by magnetron co-sputtering, J. Surf. Coat. Technol. 259 ( 2014 ) 146-151.

[2] J.C. Caicedo, G. Cabrera et al, Nature in corrosion-erosion surface for [TiN/TiAlN $]_{\mathrm{n}}$ nanometric multilayers growth on AISI 1045 steel, J. Thin Solid Film, 520 (2012) 4350-4361.

[3] Harish C. Barshilia, Moumita Ghosh, Shashidhara et al, Deposition and characterization of TiAlSiN nanocomposite coatings prepared by reactive pulsed direct current unbalanced magnetron sputtering, J. Appl. Surf. Sci. 256 (2010) 6420-6426.

[4] Hanmu Dong, Xiao Chenet al, TiN/VN composites with core/shell structure for supercapacitors, J. Mater. Res. Bull. 46 (2011) 835-839.

[5] Harish C. Barshillia, K. S. Rajam et al, A comparative study on the structure and properties of nanolayered $\mathrm{TiN} / \mathrm{NbN}$ and TiAlN/TiN multilayer coatings prepared by reactive direct current magnetron sputtering, J. Thin Solid Films. 501 (2006) 158-166.

[6] Xiaoying Li, Wenwen Wu et al, Microstructural characterisation of carbon doped CrAlTiN nanoscale multilayer coatings, J. Surf. Coat. Technol. 205 (2011) 3251-3259. 